\title{
The learning curve for minimally invasive Oxford phase 3 unicompartmental knee arthroplasty: cumulative summation test for learning curve (LC-CUSUM)
}

Qidong Zhang ${ }^{1}$, Qian Zhang ${ }^{2}$, Wanshou Guo ${ }^{1 *}$, Zhaohui Liu' ${ }^{1}$ Liming Cheng ${ }^{1}$, Debo Yue ${ }^{1}$ and Nianfei Zhang ${ }^{1}$

\begin{abstract}
Background: The minimally invasive Oxford unicompartmental knee arthroplasty (UKA) is a demanding procedure but has many advantages compared with total knee arthroplasty (TKA). The aim of this observational study was to investigate the learning curve of one experienced surgeon introducing minimally invasive Oxford phase 3 UKA into his routine clinical practice.

Methods: The first 50 consecutive cases of minimally invasive Oxford phase 3 UKA performed by one surgeon were evaluated to determine whether there was an association between outcomes and the cumulative number of cases performed, indicating the presence of learning curve. The cohort was divided into two groups: group A comprised the first 25 cases and group B cases 26-50. Duration of surgery, blood loss, Hospital for Special Surgery score, range of motion, complications, and the radiographical position of the implant were compared between the groups. The cumulative summation test for learning curve (LC-CUSUM) was then used to further analyze the learning curve.

Results: The mean age and follow-up were 64.4 years and 50.9 months, respectively. The duration of surgery and blood loss were significantly more favorable in group B. The length of incision gradually reduced from $9.7 \pm 1.3$ to $8.5 \pm 1.1 \mathrm{~cm}$. Failures were identified in nine patients (18\%). Two revisions and two dislocations were encountered in group $A$; one revision was performed 4 years after surgery for a patient in group $B$ because of a fracture. One case of lateral compartment osteoarthritis was identified in group A. Two patients in each group reported continuing unexplained pains. CUSUM analysis showed that failure rates diminished rapidly after 16 cases and reached an acceptable rate after 29 cases.
\end{abstract}

Conclusions: Minimally invasive Oxford phase 3 UKA for anteromedial osteoarthritis is a demanding procedure, but satisfactory outcomes can be achieved after approximately 25 cases.

Keywords: Unicompartmental knee arthroplasty, Total knee arthroplasty, Minimally invasive surgery, Learning curve, CUSUM analysis

\footnotetext{
* Correspondence: guowanshou@gmail.com

${ }^{1}$ Department of joint surgery, China-Japan Friendship Hospital, Yinghua

Street, Beijing 100029, People's Republic of China

Full list of author information is available at the end of the article
} 


\section{Background}

As the proportion of elderly people in the global population rises, the incidence of knee osteoarthritis is increasing. Unicompartmental knee arthroplasty (UKA) is a treatment option for patients with osteoarthritis of the medial compartment. As surgical techniques and instruments have improved, this procedure has shown many advantages over more traditional techniques, such as less soft tissue injury, a smaller incision, minimal bone resection, preservation of normal knee kinematics, reduced hospital stay, and more rapid recovery [1-4]. In addition, it could be argued that it is reasonable to undertake a UKA if osteoarthritis is only present in one compartment. The mobile Oxford medial UKA (Oxford ${ }^{\circ}$ Unicompartmental Knee, Biomet, Bridgend, UK) has been used successfully for more than three decades to treat anteromedial arthritis of the knee. The phase 3 implant was introduced in 1998 and has been widely adopted, with many reports of excellent outcomes [5-7].

A learning curve is defined as an improvement in performance over time or with increasing experience or training. The starting point of the curve in surgery indicates the baseline level of surgical skill, and the end point shows an assumed expertise level. The slope of the curve indicates the speed of learning, which may be influenced by surgeon-related factors and institutional factors, such as operating team experience, the size of the institution, caseload volume, and financial resources $[8,9]$. Most surgeons performing joint replacements had a great deal of experience in total knee arthroplasty (TKA) before UKA procedure; however, the surgical principles, techniques, and management of UKA and TKA are different and UKA can be very challenging. Good clinical practice and the introduction of new orthopedic techniques mandate the need to determine the nature of the learning curve. The cumulative summation method (CUSUM) is a sequential analysis tool that was initially used in industrial settings for quality control purposes. It can be used to establish the learning curve for a surgical procedure and allows one to judge when an individual's performance has achieved a predefined level of competence. The aim of this study was to establish the learning curve of an experienced knee surgeon introducing minimally invasive Oxford phase 3 UKA into routine clinical practice using the CUSUM technique.

\section{Methods}

Approval of the study was obtained from the institutional review board. From January 2009 to March 2010, 50 consecutive UKA cases performed by senior author were included in the analysis, with no loss to follow-up.

The indication for UKA was anteromedial osteoarthritis with severe medial knee pain and radiographic evidence of osteoarthritis in the medial compartment. Other indications were an intact anterior cruciate ligament (ACL), varus deformity $<15^{\circ}$, flexion contracture $<15^{\circ}$, and an intact lateral compartment [10]. The preoperative diagnosis was primary anteromedial osteoarthritis in all patients.

All patients were placed in the supine position on a standard operating table after spinal anesthesia had been administered. A tourniquet was applied to the proximal thigh on the operative side and inflated to $300 \mathrm{~mm} \mathrm{Hg}$. A medial parapatellar incision was used, and the patella was not everted. Non-anatomic bearings were used in all cases.

Clinical outcomes were evaluated by measuring the difference between the range of knee motion (ROM) and Hospital for Special Surgery (HSS) knee score before surgery and at final follow-up. Weight-bearing anteroposterior and lateral radiographs of the knee were obtained, as well as a long hip-to-ankle film to assess the femorotibial angle and implant position. Loosening of the tibial or femoral components was identified by an area of radiolucency $>2 \mathrm{~mm}$ around the components. Overrotation of the component was diagnosed if the alignment angle exceeded $10^{\circ}$. Each evaluation was made twice by two independent observers. The end point for survival was defined as revision for any reason. Patient-related information was collected using a standardized questionnaire administered before surgery and at follow-up.

\section{Statistical analysis}

All data were analyzed using SPSS version 17.0 (SPSS Inc., Chicago, IL, USA). Data are reported as the mean with the standard deviation. The chi-squared test and Student's $t$ test were used to determine whether there were statistically significant differences between the groups. A $p$ value $<0.05$ was considered statistically significant [11].

\section{CUSUM analysis}

For CUSUM analysis, four parameters are defined: the acceptable failure rate $(p 0)$, the unacceptable failure rate $(p 1)$, the type I error rate $(\alpha)$, and the type II error rate $(\beta)$. The equations shown in Table 1 are used to calculate the CUSUM score [12]. On the basis of a literature review, we defined the acceptable UKA failure rate as $20 \%$ and the unacceptable failure rate as $40 \%[2,13]$. The probabilities of $\alpha$ and $\beta$ were set at 0.05 and 0.20 , respectively. Failure situations were defined as revision, reoperation, postoperative complications, and radiographic malposition. The results of CUSUM analysis are presented in a chart with case numbers plotted on the $x$ axis and the corresponding CUSUM score on the $y$-axis, which allows performance over consecutive procedures to be visualized. When a failure occurred, the constant ' $1-S$ ' was added to the cumulative score. When a 
Table 1 CUSUM equations and variables

\begin{tabular}{ll}
\hline Variable & Value \\
\hline$p 0$-acceptable UKA failure rate & 0.20 \\
$p 1$-unacceptable UKA failure rate & 0.40 \\
$a$-probability of the type I error & 0.05 \\
$\beta$-probability of the type II error & 0.20 \\
$P=\ln (p 1 / p 0)$ & 0.69 \\
$Q=\ln [(1-p 0) /(1-p 1)]$ & 0.29 \\
$S=Q /(P+Q)$ & 0.29 \\
$1-S$ & 0.71 \\
$a=\ln [(1-\beta) / a]$ & 2.77 \\
$b=\ln [(1-a) / \beta]$ & 1.56 \\
$h 0=-b /(P+Q)$ & -1.59 \\
$h 1=a /(P+Q)$ & 2.83 \\
\hline
\end{tabular}

success occurred, the variable ' $s$ ' was subtracted from the cumulative score. Hence, success is rewarded by a downward slope whereas failure is represented by an upward slope on the chart. If the line crosses the upper decision limit (h1) from below, this indicates that the actual failure rate is equal to the unacceptable failure rate with a type I error. If the line crosses the lower decision limit (h0) from above, this indicates that the actual failure rate does not differ from the acceptable failure rate with a type II error probability of 0.20 . When the line is between h1 and h0, no statistical inference can be made.

\section{Results}

One patient in group A died from lung cancer 34 months after surgery, but there were no symptoms or clinical signs of implant failure or radiographic signs of loosening at the last follow-up. The mean follow-up was $50.9 \pm$ 4.9 months (range 45-60 months) after the final assessment in December 2013. The mean age on the day of surgery was $64.4 \pm 8.5$ years. Twenty-seven UKAs were performed on the right knee and 23 on the left; 16 patients were male and 34 female. There were no significant differences in any of the clinical characteristics of the patients in each group.

The mean duration of surgery decreased with the number of cumulative cases, from $85.0 \pm 15.7 \mathrm{~min}$ in group $\mathrm{A}$ to $64.4 \pm 13.9 \mathrm{~min}$ in group $\mathrm{B}(p<0.05)$. Perioperative blood loss was also lower in group B (185.0 \pm $69.2 \mathrm{ml}$ compared with $226.2 \pm 74.8 \mathrm{ml}$ in group $\mathrm{A} ; p<$ $0.05)$. The mean incision length was $9.7 \pm 1.3 \mathrm{~cm}$ in group A compared with $8.5 \pm 1.1 \mathrm{~cm}$ in group $\mathrm{B}$ ( $p=$ $0.001)$. In all patients, passive full flexion of the knee and painless active full flexion was possible within 7 postoperative days and 3 postoperative months, respectively. The mean preoperative $\mathrm{ROM}$ was $125.0^{\circ} \pm 9.1^{\circ}$, which improved to a mean of $126.6^{\circ} \pm 8.0^{\circ}$ at final follow-up, although this difference was not statistically significant $(p=0.349)$. The mean HSS score increased from $59.5 \pm 8.9$ to $88.3 \pm 9.3$ at the time of final followup $(p=0.000)$. Postoperative HSS scores were significantly higher in group B $(92.4 \pm 4.5$ compared with $84.3 \pm 11.1$ in group A, $p=0.002$; Table 2).

The CUSUM learning curve is shown in Figure 1 and Table 3. Failures were identified in nine patients (18\%). There was a trend toward a higher failure rate during the first 25 cases, although this did not achieve statistical significance $(28 \%$ in group A compared with $8 \%$ in group B; $p=0.138)$. Three of the 50 prostheses $(6 \%)$ included in the analysis had been revised before final follow-up; with the end point of revision for any reason, the survival rate was $94 \%$. In group A, one UKA was revised to a TKA as a consequence of aseptic loosening of the tibial component 3.5 years after surgery and one was revised to a TKA due to infection 1 year after surgery. In group $\mathrm{B}$, one UKA was revised to a TKA after 4 years as a result of a lateral tibial plateau fracture sustained in a major trauma. There had been no clinical symptoms of implant failure or radiographic signs of loosening before the accident. Bearing dislocation occurred in two cases 1.5 and 2 years after surgery because of laxity after hyperflexion trauma; the bearings were replaced by thicker ones. No recurrences of luxation were seen at follow-up. One diagnosis of lateral compartment osteoarthritis was made in group A (Figures 2, 3, 4). Two patients in each group reported continuing unexplained pains. There were no serious adverse events, such as pulmonary embolism, deep venous thrombosis, or iatrogenic neurovascular injury. According to the guidelines proposed by the Oxford group [14], postoperative radiographic assessments showed that one of the components in group A was not in an acceptable position.

The CUSUM learning curve chart (Figure 1) shows that case 16 corresponds to the main inflection point (point A) at which the failure rate became consistent. At

Table 2 UKA Demographics

\begin{tabular}{llll}
\hline & $\mathbf{A}(\boldsymbol{N}=\mathbf{2 5})$ & $\mathbf{B}(\boldsymbol{N}=\mathbf{2 5})$ & $\boldsymbol{p}$ value \\
\hline Age (years) & $62.8 \pm 9.4$ & $66.1 \pm 7.2$ & 0.168 \\
Sex (male/female) & $9: 16$ & $7: 18$ & 0.544 \\
Side (right/left) & $12: 13$ & $15: 10$ & 0.395 \\
BMI (kg/m $\left.{ }^{2}\right)$ & $24.9 \pm 1.3$ & $23.8 \pm 4.1$ & 0.316 \\
Duration of surgery (min) & $85.0 \pm 15.7$ & $64.4 \pm 13.9$ & 0.000 \\
Length of incision (cm) & $9.7 \pm 1.3$ & $8.5 \pm 1.1$ & 0.001 \\
Blood loss (ml) & $226.2 \pm 74.8$ & $185.0 \pm 69.2$ & 0.049 \\
Preoperative HSS score & $57.8 \pm 8.5$ & $61.2 \pm 9.1$ & 0.189 \\
Preoperative range of motion & $126.3 \pm 7.6$ & $123.7 \pm 10.3$ & 0.306 \\
Postoperative HSS score & $84.3 \pm 11.1$ & $92.4 \pm 4.5$ & 0.002 \\
Postoperative range of motion & $125.0 \pm 9.0$ & $128.2 \pm 6.7$ & 0.160 \\
\hline
\end{tabular}




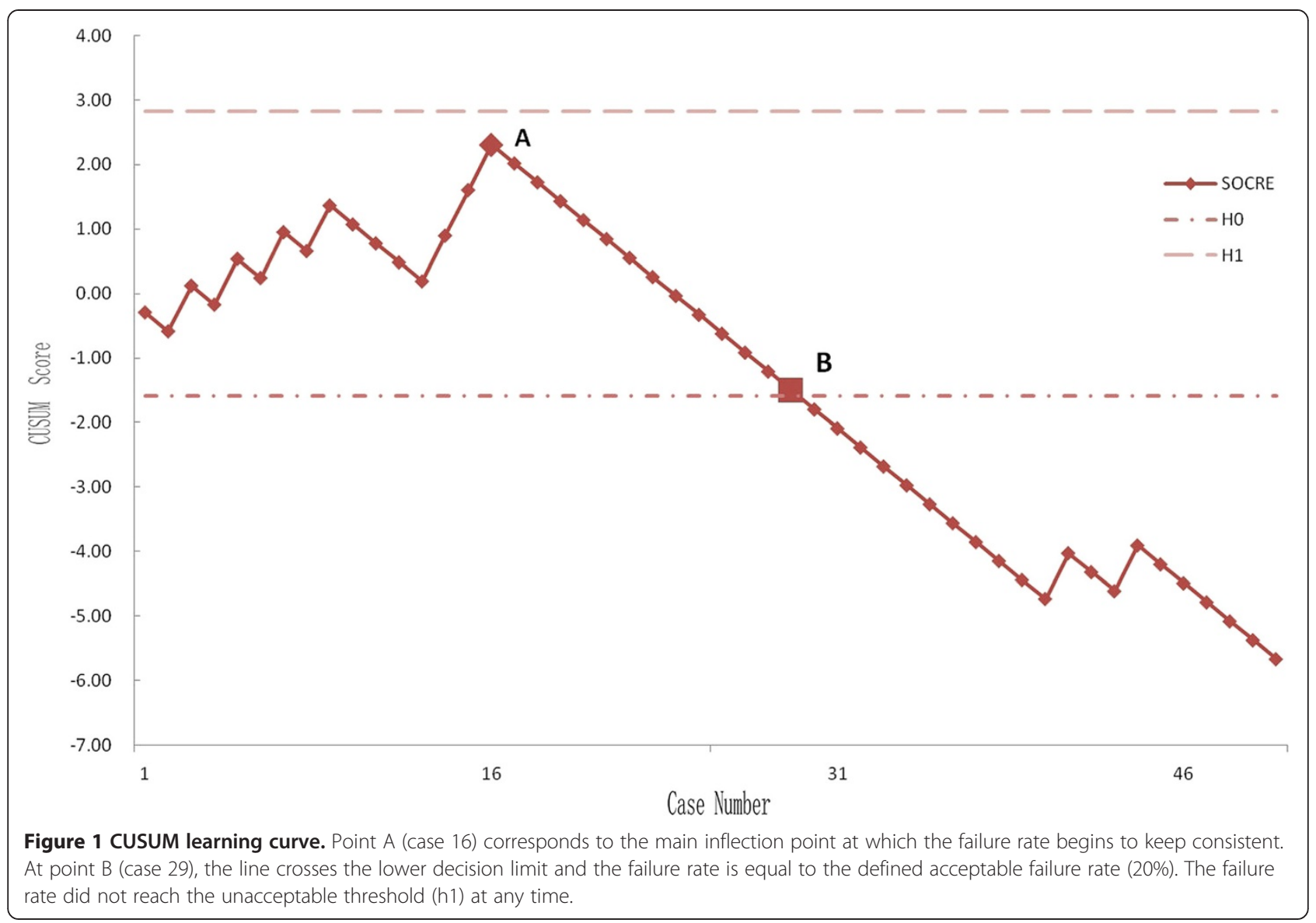

point B (case 29), the line crosses the lower decision limit and the failure rate is equal to the defined acceptable failure rate $(20 \%)$. The failure rate did not reach the unacceptable threshold (h1) at any time.

\section{Discussion}

Unicompartmental arthroplasty is a well-recognized treatment option for unicompartmental osteoarthritis of the knee. The Oxford unicompartmental knee has a mobile bearing with full congruency, minimizing polyethylene wear, and there are many reports of excellent postoperative outcomes [2,3,15-17]. Svard and Price reported a 95\% cumulative survival rate over 10 years [4]. Pandit et al. reported the outcomes of 1,000 phase 3 Oxford medial UKAs using a minimally invasive surgical approach by two surgeons. Using revision as the end point, the 10-year survival rate was 99.8\% [18]. In 2011, Price et al. reported the second decade data of the Oxford UKA, having previously reported longitudinal data from 1,6 , and 10 postoperative years $[15,19,20]$. In the most recent study, postoperative function and HSS score had still significantly improved from baseline. These findings suggest that the Oxford UKA is a reliable treatment option for anteromedial osteoarthritis of the knee.

Despite these apparent advantages, other investigators have reported less favorable long-term results or early failures that have required revision or reoperation [21-24]. The minimally invasive medial Oxford UKA is undoubtedly a demanding procedure, and many early failures are often due to technical errors [25]. Kort and colleagues [5] assessed 130 patients with minimally invasive Oxford phase 3 UKAs over a follow-up period of 2-7 years and reported an overall survival rate of $89 \%$. Of 17 failures, they attributed 13 to human error, confirming that surgical expertise influences mid-term results and thereby long-term outcomes. Similarly, Kuipers and colleagues [26] reported that the cumulative survival rate of a cohort of 437 Oxford phase 3 implants was $84.7 \%$ at 5 years and that 101 of these were at risk. Each surgeon in the study performed an average of eight UKAs per year. In 2001, Robertson reported that centers that performed fewer than 23 UKAs annually had a revision risk 1.63 times higher than centers with higher volumes of casework [27]. Surgical experience therefore appears to be a critical determinant of outcome. These results also underline the 
Table 3 CUSUM chart calculations

\begin{tabular}{|c|c|c|c|c|}
\hline Case & Sex & Age (year) & CUSUM score & Failure model \\
\hline 1 & M & 60 & -0.29 & \\
\hline 2 & $\mathrm{~F}$ & 57 & -0.59 & \\
\hline 3 & $\mathrm{~F}$ & 55 & 0.12 & Unexplained pain \\
\hline 4 & M & 76 & -0.17 & \\
\hline 5 & $\mathrm{~F}$ & 49 & 0.53 & Revision for infection \\
\hline 6 & M & 52 & 0.24 & \\
\hline 7 & $\mathrm{~F}$ & 50 & 0.95 & $\begin{array}{l}\text { Revision for } \\
\text { aseptic loosening }\end{array}$ \\
\hline 8 & $\mathrm{~F}$ & 67 & 0.65 & \\
\hline 9 & $\mathrm{~F}$ & 71 & 1.36 & Malposition \\
\hline 10 & $\mathrm{~F}$ & 52 & 1.07 & \\
\hline 11 & M & 75 & 0.77 & \\
\hline 12 & $\mathrm{~F}$ & 56 & 0.48 & \\
\hline 13 & $\mathrm{~F}$ & 54 & 0.19 & \\
\hline 14 & $\mathrm{~F}$ & 61 & 0.89 & Bearing dislocation \\
\hline 15 & $\mathrm{~F}$ & 61 & 1.60 & $\begin{array}{l}\text { Lateral compartment } \\
\text { degeneration }\end{array}$ \\
\hline 16 & $\mathrm{~F}$ & 54 & 2.31 & Bearing dislocation \\
\hline 17 & M & 74 & 2.01 & \\
\hline 18 & M & 70 & 1.72 & \\
\hline 19 & $\mathrm{~F}$ & 72 & 1.43 & \\
\hline 20 & M & 73 & 1.13 & \\
\hline 21 & $\mathrm{~F}$ & 69 & 0.84 & \\
\hline 22 & M & 70 & 0.55 & \\
\hline 23 & F & 70 & 0.25 & \\
\hline 24 & $M$ & 57 & -0.04 & \\
\hline 25 & $\mathrm{~F}$ & 79 & -0.33 & \\
\hline 26 & $\mathrm{~F}$ & 65 & -0.63 & \\
\hline 27 & $\mathrm{~F}$ & 75 & -0.92 & \\
\hline 28 & F & 72 & -1.21 & \\
\hline 29 & M & 61 & -1.51 & \\
\hline 30 & M & 59 & -1.80 & \\
\hline 31 & $\mathrm{~F}$ & 61 & -2.09 & \\
\hline 32 & M & 64 & -2.39 & \\
\hline 33 & M & 64 & -2.68 & \\
\hline 34 & F & 57 & -2.97 & \\
\hline 35 & $\mathrm{~F}$ & 76 & -3.27 & \\
\hline 36 & $\mathrm{~F}$ & 82 & -3.56 & \\
\hline 37 & F & 67 & -3.85 & \\
\hline 38 & F & 62 & -4.15 & \\
\hline 39 & $\mathrm{~F}$ & 71 & -4.44 & \\
\hline 40 & $\mathrm{~F}$ & 56 & -4.73 & \\
\hline 41 & M & 57 & -4.03 & Fracture \\
\hline 42 & $\mathrm{~F}$ & 70 & -4.32 & \\
\hline 43 & $M$ & 62 & -4.61 & \\
\hline
\end{tabular}

Table 3 CUSUM chart calculations (Continued)

\begin{tabular}{lllll}
\hline 44 & $F$ & 55 & -3.91 & Unexplained pain \\
45 & $F$ & 78 & -4.20 & \\
46 & $F$ & 58 & -4.49 & \\
47 & $F$ & 73 & -4.79 & \\
48 & $F$ & 61 & -5.08 & \\
49 & $F$ & 70 & -5.37 & \\
50 & $M$ & 61 & -5.67 & \\
\hline
\end{tabular}

existence of a learning curve for UKA and that minimally invasive Oxford phase 3 UKA is a demanding procedure that nonetheless has the potential to achieve satisfying surgical outcomes.

In our study, the five patients who required reoperations were all young: their ages at operation were 49, 50, 54, 57, and 61 years. Young patients are more likely to be physically active after surgery, which may be a risk factor for failure [26,28]. The patient in group B who was not satisfied with her outcome was of perimenopausal age, and it is possible that patients with endocrinesensitive disease might not achieve such favorable surgical outcomes. It is very important that patients have realistic expectations about the extent of pain relief, the amount of physical activity permitted after surgery, and the likely

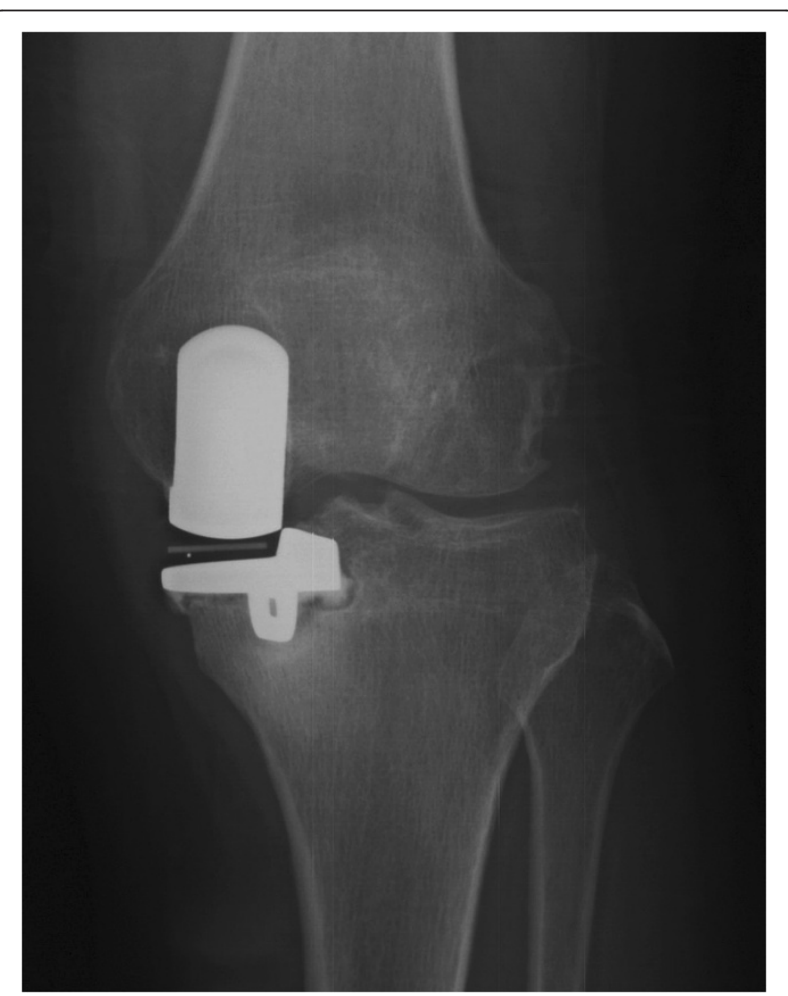

Figure 2 Anteroposterior X-ray of postoperative UKA infection. 


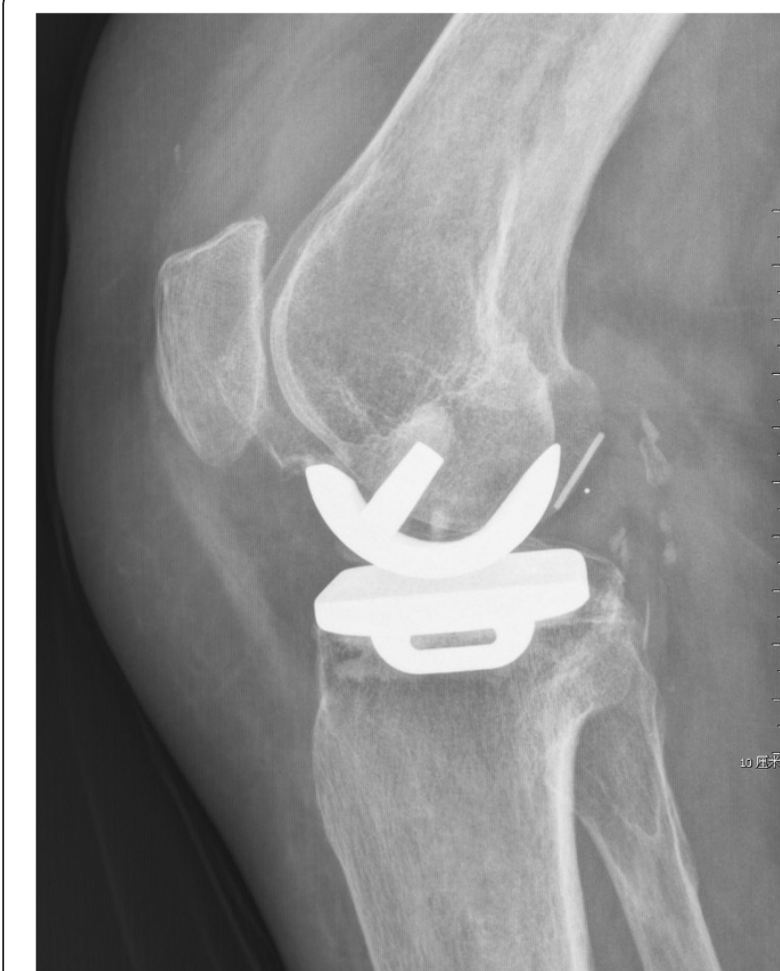

Figure 3 Posterior dislocation of the bearing.

duration of recovery following UKA in order to achieve high satisfaction rates.

As with the slope of the learning curve, the duration of surgery, blood loss, and length of incision gradually improved with the cumulative number of procedures. Nonetheless, we experienced many challenges due to the complexity of surgery. In summary, we found that it is essential (but may be difficult) to equalize the $90^{\circ}$ and $20^{\circ}$ flexion gaps. The principles of correct alignment and joint stability that are so important for TKA are likely to be at the forefront of the surgeon's mind. To achieve stability, we elected to create a small tight extension gap in the early cases, which likely accounts for the one case of overcorrection in group A. Overcorrection of the tibiofemoral deformity can lead to failure because of degenerative change in the contralateral compartment [29]. Concern about this potential complication meant that we changed our practice so that a small but loose extension gap was acceptable. Undercorrection with a smaller bearing can cause bearing dislocation and increase the load to the medial compartment, which may accelerate polyethylene wear [25]. In our early cases, two bearing dislocations were encountered because of laxity, which might have been due to incomplete gap balancing between flexion and extension. In our cases of bearing dislocation, the bearings were replaced by new thicker ones. Although no recurrences of luxation occurred, osteoarthritis may

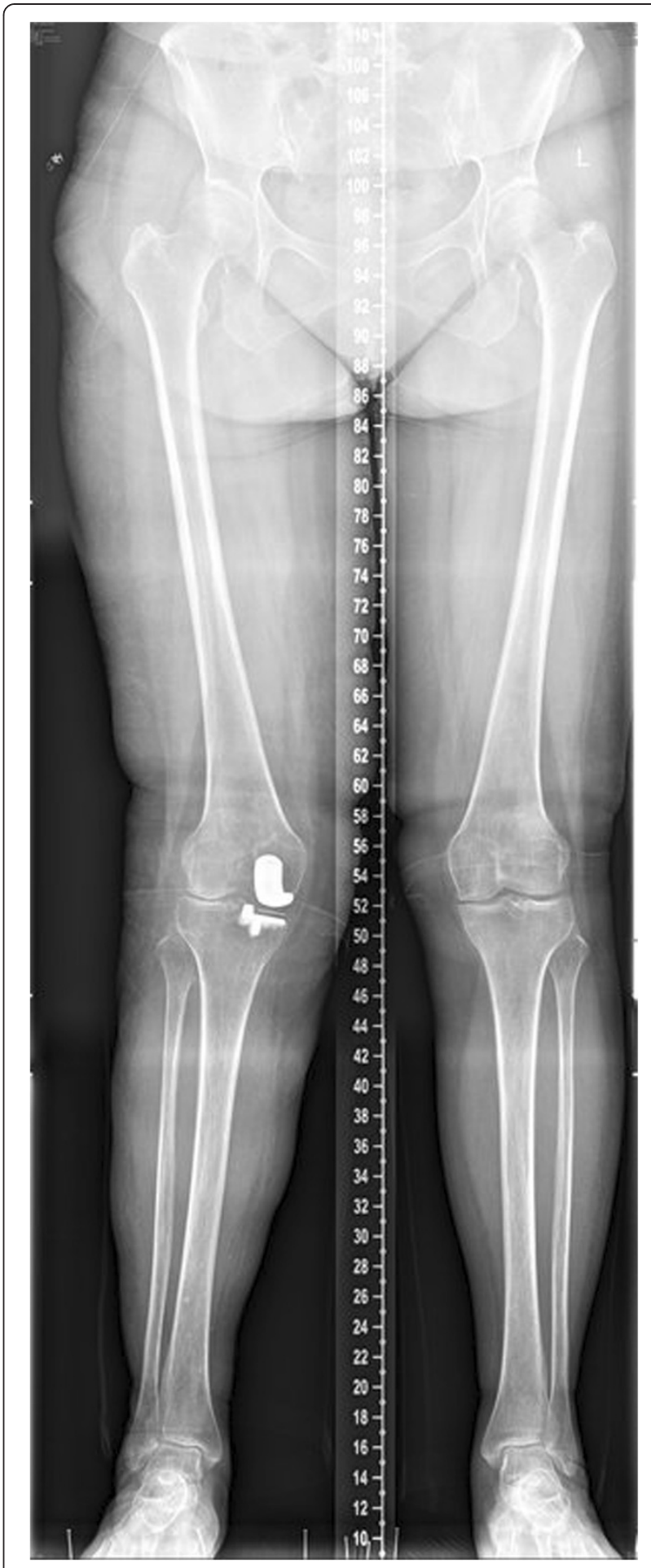

Figure 4 Lateral compartment degeneration with valgus malalignment status after UKA.

progress in the lateral compartment in the future due to overcorrection of the alignment. A longer-term study is needed to examine this issue. 
One essential surgical objective is accurate positioning of the components according to the guidelines proposed by the Oxford group [14]. There are many factors that must be assessed on the postoperative radiographs. The tibial component should be positioned just medial to the apex of the medial spine and should extend to the medial margin of the tibia or overhang by up to $2 \mathrm{~mm}$. It

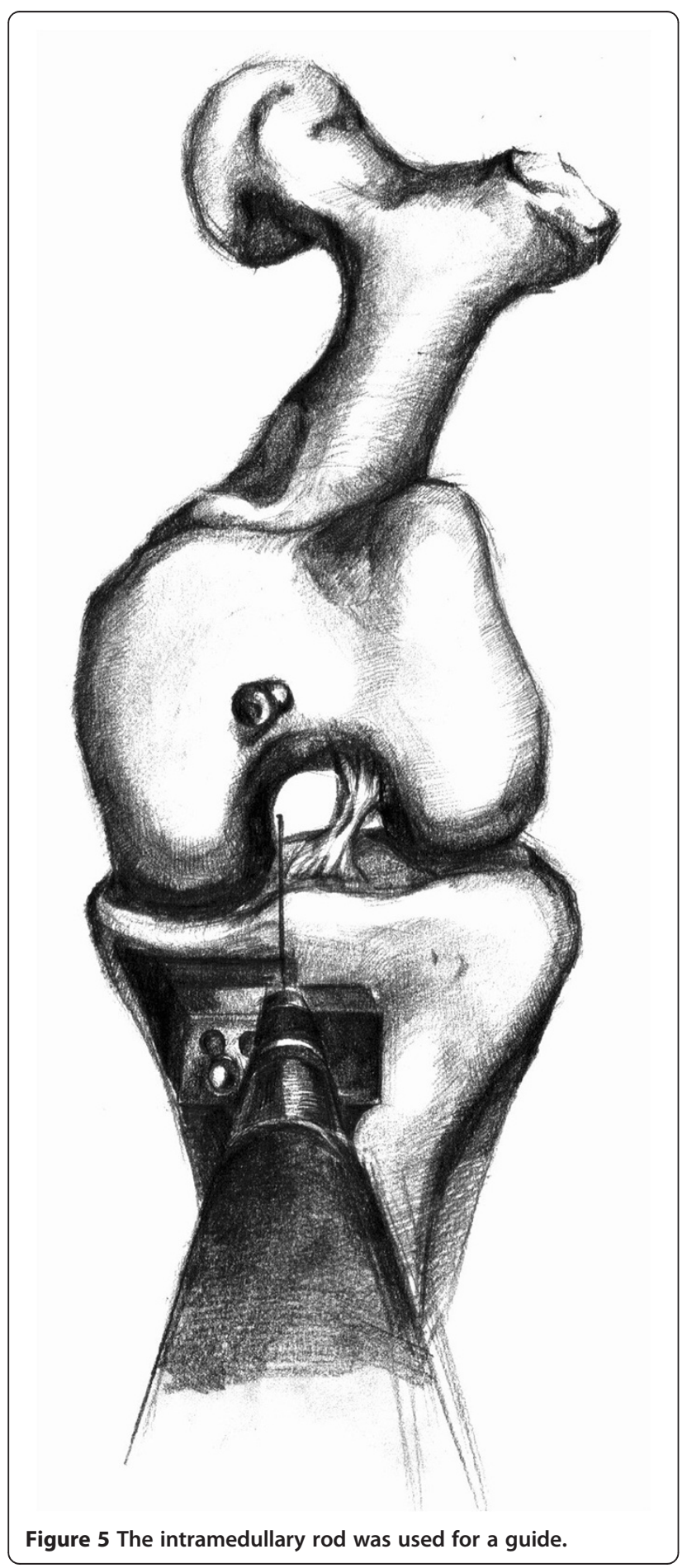

should also reach the posterior cortex of the tibia. The bearing should be a few millimeters away from the vertical wall. Malalignment of the femoral component of up to $10^{\circ}$ and of the tibial component of up to $5^{\circ}$ is considered acceptable. In the earlier cases, we found the vertical bone cut in the tibial plateau challenging. The Oxford group's guidelines state that the blade should point to the head of the femur; however, we found it

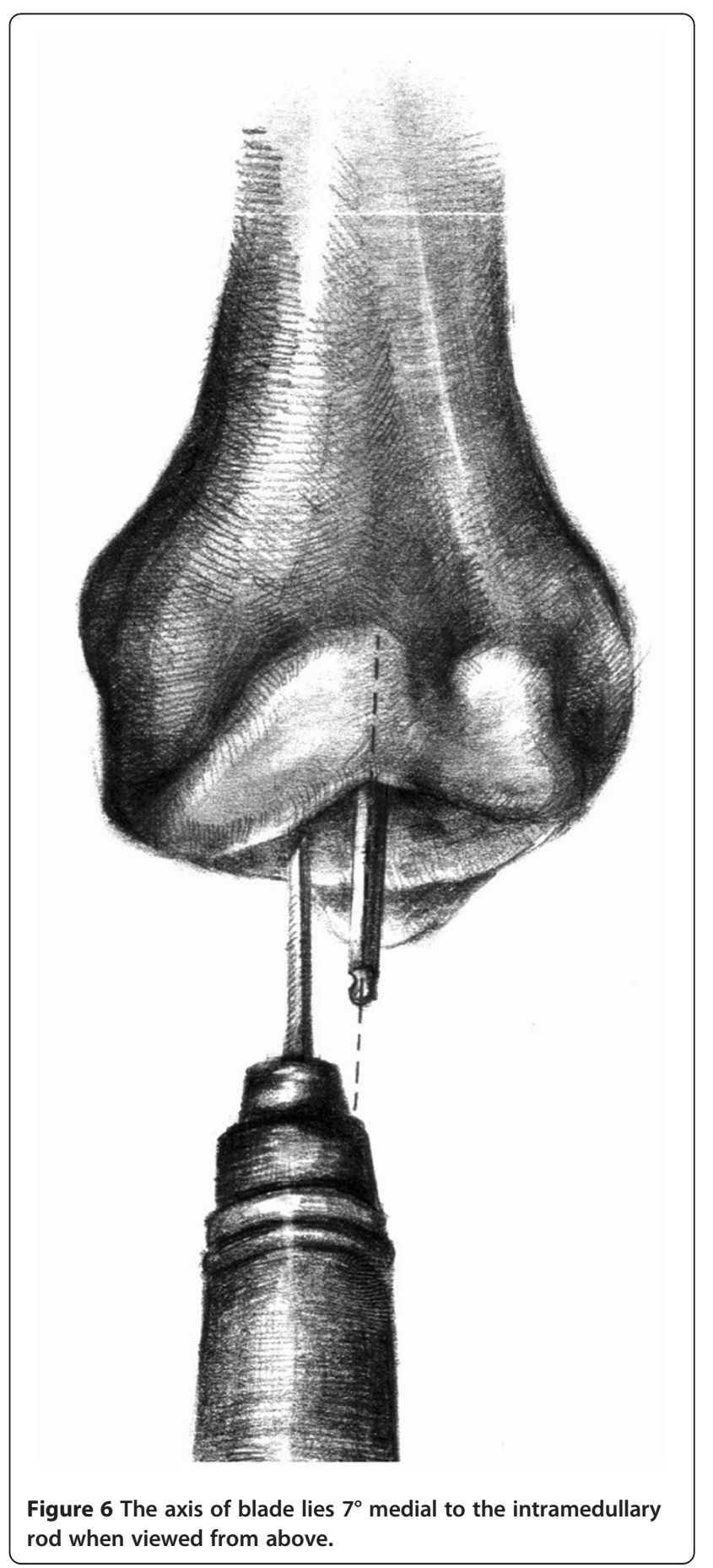


difficult to locate the position of the head of the femur accurately. Consequently, postoperative radiographs taken to assess implant positioning showed that one implant was not within the recommended limits for positioning criteria. We modified our technique and used the intramedullary rod as a guide. The axis of the blade lay $7^{\circ}$ medial to the intramedullary rod when viewed from above (Figures 5 and 6). With this technique, the number of malposition was reduced.

Our study has some limitations. First, by virtue of this type of study, the follow-up time of group B was shorter than that of group A, which may have influenced our ability to detect some longer-term complications. Second, we examined the outcomes of a single experienced knee surgeon, so our findings may not be representative of surgeons with other levels of expertise. Third, the follow-up time was relatively short, and a longer-term study is needed to confirm our results.

\section{Conclusions}

Minimally invasive Oxford phase 3 UKA for medial unicompartmental knee osteoarthritis is a demanding procedure but only needs a short learning curve to achieve satisfactory surgical outcomes in the hands of an experienced knee surgeon. The mean duration of surgery, incision length, blood loss, and the incidence of postoperative complications decline with experience. Based on our CUSUM analysis, 25 cases are required before a consistently low failure rate is achieved for minimally invasive Oxford phase 3 UKA.

\section{Abbreviations \\ UKA: unicompartmental knee arthroplasty; LC-CUSUM: cumulative summation test for learning curve; TKA: total knee arthroplasty; HSS: Hospital for special surgery; ACL: anterior cruciate ligament.}

\section{Competing interests}

The authors declare that they have no competing interests.

\section{Authors' contributions}

QDZ and WSG conceived of the study. QDZ and QZ carried out the data collection and performed the statistical analysis and drafted the manuscript. ZHL, LMC, DBY, and NFZ also carried out the data collection. ZHL and LMC participated in the design and coordination and helped draft the manuscript. All authors read and approved the final manuscript.

\section{Acknowledgements}

We thank Lei Wang for his support to draw diagram for this study.

\section{Author details}

'Department of joint surgery, China-Japan Friendship Hospital, Yinghua Street, Beijing 100029, People's Republic of China. ${ }^{2}$ Beijing University of Chinese Medicine, Yinghua Street, Beijing 100029, People's Republic of China.

Received: 11 May 2014 Accepted: 22 August 2014

Published: 6 September 2014

\section{References}

1. Berger RA, Della VC: Unicompartmental knee arthroplasty: indications, techniques, and results. Instr Course Lect 2010, 59:47-56.

2. Lisowski LA, van den Bekerom MP, Pilot P, van Dijk CN, Lisowski AE: Oxford Phase 3 unicompartmental knee arthroplasty: medium-term results of a minimally invasive surgical procedure. Knee Surg Sports Traumatol Arthrosc 2011, 19:277-284.

3. Price AJ, Dodd CA, Svard UG, Murray DW: Oxford medial unicompartmental knee arthroplasty in patients younger and older than 60 years of age. J Bone Joint Surg (Br) 2005, 87:1488-1492.

4. Svard UC, Price AJ: Oxford medial unicompartmental knee arthroplasty. A survival analysis of an independent series. J Bone Joint Surg (Br) 2001, 83:191-194.

5. Kort NP, van Raay JJ, van Horn JJ: The Oxford phase III unicompartmental knee replacement in patients less than 60 years of age. Knee Surg Sports Traumatol Arthrosc 2007, 15:356-360.

6. Pandit $\mathrm{H}$, Jenkins C, Barker K, Dodd CA, Murray DW: The Oxford medial unicompartmental knee replacement using a minimally-invasive approach. J Bone Joint Surg (Br) 2006, 88:54-60.

7. Tang $H$, Zhao L, Yan H, Jin D, Su X: Mid-term effectiveness of Oxford Unicompartmental Knee System Phase III for medial unicompartmental knee osteoarthritis. Zhongguo Xiu Fu Chong Jian Wai Ke Za Zhi 2012, 26:17-20.

8. Dionigi G, Bacuzzi A, Boni L, Rovera F, Dionigi R: What is the learning curve for intraoperative neuromonitoring in thyroid surgery? Int J Surg 2008, 6(Suppl 1):S7-S12.

9. Walton $\mathrm{R}$, Theodorides A, Molloy A, Melling D: Is there a learning curve in foot and ankle surgery? Foot Ankle Surg 2012, 18:62-65.

10. Murray DW: Mobile bearing unicompartmental knee replacement. Orthopedics 2005, 28:985-987.

11. Nayak BK, Hazra A: How to choose the right statistical test? Indian J Ophthalmol 2011, 59:85-86.

12. Biau DJ, Porcher R: A method for monitoring a process from an out of control to an in control state: application to the learning curve. Stat Med 2010, 29:1900-1909.

13. Lee YK, Ha YC, Hwang DS, Koo KH: Learning curve of basic hip arthroscopy technique: CUSUM analysis. Knee Surg Sports Traumatol Arthrosc 2013, 21:1940-1944.

14. Goodfellow JW, O'Connor J, Dodd CAF, Murray DW: Unicompartmental arthroplasty with the Oxford knee. Oxford: Oxford University Press; 2006:117-128.

15. Price AJ, Svard U: A second decade lifetable survival analysis of the Oxford unicompartmental knee arthroplasty. Clin Orthop Relat Res 2011, 469:174-179.

16. Emerson RJ, Higgins LL: Unicompartmental knee arthroplasty with the oxford prosthesis in patients with medial compartment arthritis. J Bone Joint Surg Am 2008, 90:118-122.

17. Kim KT, Lee S, Park HS, Cho KH, Kim KS: A prospective analysis of Oxford phase 3 unicompartmental knee arthroplasty. Orthopedics 2007, 30:15-18.

18. Pandit H, Jenkins C, Gill HS, Barker K, Dodd CA, Murray DW: Minimally invasive Oxford phase 3 unicompartmental knee replacement: results of 1000 cases. J Bone Joint Surg (Br) 2011, 93:198-204.

19. Price AJ, Short A, Kellett C, Beard D, Gill H, Pandit H, Dodd CA, Murray DW: Ten-year in vivo wear measurement of a fully congruent mobile bearing unicompartmental knee arthroplasty. J Bone Joint Surg (Br) 2005, 87:1493-1497.

20. Price AJ, Waite JC, Svard U: Long-term clinical results of the medial Oxford unicompartmental knee arthroplasty. Clin Orthop Relat Res 2005, 435:171-180.

21. Edmondson MC, Isaac D, Wijeratna M, Brink S, Gibb P, Skinner P: Oxford unicompartmental knee arthroplasty: medial pain and functional outcome in the medium term. J Orthop Surg Res 2011, 6:52.

22. Munk S, Odgaard A, Madsen F, Dalsgaard J, Jorn LP, Langhoff O, Jepsen CF, Hansen TB: Preoperative lateral subluxation of the patella is a predictor of poor early outcome of Oxford phase-III medial unicompartmental knee arthroplasty. Acta Orthop 2011, 82:582-588.

23. Rea P, Short A, Pandit H, Price AJ, Kyberd P, Beard DJ, Gill HS, Murray DW: Radiolucency and migration after Oxford unicompartmental knee arthroplasty. Orthopedics 2007, 30:24-27.

24. Saldanha KA, Keys GW, Svard UC, White SH, Rao C: Revision of Oxford medial unicompartmental knee arthroplasty to total knee arthroplasty results of a multicentre study. Knee 2007, 14:275-279.

25. Lewold S, Goodman S, Knutson K, Robertsson O, Lidgren L: Oxford meniscal bearing knee versus the Marmor knee in unicompartmental arthroplasty for arthrosis. A Swedish multicenter survival study. J Arthroplasty 1995, 10:722-731. 
26. Kuipers BM, Kollen BJ, Bots PC, Burger BJ, van Raay JJ, Tulp NJ, Verheyen CC: Factors associated with reduced early survival in the Oxford phase III medial unicompartment knee replacement. Knee 2010, 17:48-52.

27. Robertsson O, Knutson K, Lewold S, Lidgren L: The routine of surgical management reduces failure after unicompartmental knee arthroplasty. J Bone Joint Surg (Br) 2001, 83:45-49.

28. W-Dahl A, Robertsson O, Lidgren L, Miller L, Davidson D, Graves S: Unicompartmental knee arthroplasty in patients aged less than 65 . Acta Orthop 2010, 81:90-94.

29. Padgett $D E$, Stern $S H$, Insall JN: Revision total knee arthroplasty for failed unicompartmental replacement. J Bone Joint Surg Am 1991, 73:186-190.

doi:10.1186/s13018-014-0081-8

Cite this article as: Zhang et al:: The learning curve for minimally invasive Oxford phase 3 unicompartmental knee arthroplasty:

cumulative summation test for learning curve (LC-CUSUM). Journal of Orthopaedic Surgery and Research 2014 9:81.

\section{Submit your next manuscript to BioMed Central and take full advantage of:}

- Convenient online submission

- Thorough peer review

- No space constraints or color figure charges

- Immediate publication on acceptance

- Inclusion in PubMed, CAS, Scopus and Google Scholar

- Research which is freely available for redistribution 